\title{
Proteomic Adaptation of Streptococcus pneumoniae to the Human Antimicrobial Peptide LL-37
}

\author{
Pierre-Alexander Mücke ${ }^{1}$, Sandra Maaß ${ }^{1}{ }^{(0)}$, Thomas P. Kohler ${ }^{2}{ }^{(D}$, Sven Hammerschmidt ${ }^{2}{ }^{(1)}$ and \\ Dörte Becher 1,*(1) \\ 1 Department of Microbial Proteomics, Institute of Microbiology, Center for Functional Genomics of Microbes, \\ University of Greifswald, Felix-Hausdorff-Str. 8, 17489 Greifswald, Germany; \\ pierre.muecke@uni-greifswald.de (P.-A.M.); sandra.maass@uni-greifswald.de (S.M.) \\ 2 Department of Molecular Genetics and Infection Biology, Interfaculty Institute for Genetics and Functional \\ Genomics, Center for Functional Genomics of Microbes, University of Greifswald, Felix-Hausdorff-Str. 8, \\ 17489 Greifswald, Germany; kohlert@uni-greifswald.de (T.P.K.); \\ sven.hammerschmidt@uni-greifswald.de (S.H.) \\ * Correspondence: dbecher@uni-greifswald.de; Tel.: +49-3834-420-5903
}

Received: 22 January 2020; Accepted: 10 March 2020; Published: 14 March 2020

\begin{abstract}
Secreted antimicrobial peptides (AMPs) are an important part of the human innate immune system and prevent local and systemic infections by inhibiting bacterial growth in a concentration-dependent manner. In the respiratory tract, the cationic peptide LL-37 is one of the most abundant AMPs and capable of building pore complexes in usually negatively charged bacterial membranes, leading to the destruction of bacteria. However, the adaptation mechanisms of several pathogens to LL-37 are already described and are known to weaken the antimicrobial effect of the AMP, for instance, by repulsion, export or degradation of the peptide. This study examines proteome-wide changes in Streptococcus pneumoniae D39, the leading cause of bacterial pneumonia, in response to physiological concentrations of LL-37 by high-resolution mass spectrometry. Our data indicate that pneumococci may use some of the known adaptation mechanisms to reduce the effect of LL-37 on their physiology, too. Additionally, several proteins seem to be involved in resistance to AMPs which have not been related to this process before, such as the teichoic acid flippase TacF (SPD_1128). Understanding colonization- and infection-relevant adaptations of the pneumococcus to AMPs, especially LL-37, could finally uncover new drug targets to weaken the burden of this widespread pathogen.
\end{abstract}

Keywords: Streptococcus pneumoniae; antimicrobial peptide; LL-37; adaptation; proteomics

\section{Introduction}

Infections of the lower respiratory tract, especially pneumonia, remain the third leading cause of death and the deadliest communicable disease [1]. Among the causative agents of these diseases is the Gram-positive bacteria Streptococcus pneumoniae, the leading cause of bacterial pneumonia [2]. Besides that, S. pneumoniae is responsible for other serious diseases like meningitis and septicemia following bacterial invasion into the bloodstream and milder, but even more frequent infections, of the middle ear (otitis media) or sinuses (sinusitis) [3].

Today, two main approaches are used to fight pneumococcal disease. First, vaccination to prevent initial infection, and second, treatment with classical antibiotics. However, the currently available vaccines against $S$. pneumoniae are solely directed against capsule serotypes of selected strains, and debate about the rapid replacement of vaccine-serotypes with non-vaccine-serotypes is rising [4]. On the other hand, the permanent risk for the development of antibiotic-resistant pneumococcal strains 
makes it necessary to develop new antimicrobial strategies to combat S. pneumoniae infections in the future [5].

Interestingly, humans themselves produce endogenous antibiotics called antimicrobial peptides (AMPs) to inhibit infections [6]. In general, antimicrobial peptides are small (12-50 amino acids) amphipathic molecules that exhibit a net-positive charge under physiological conditions [7,8]. Specificity for the bacterial membranes is mainly due to anionic charged phospholipids, in addition to negative charges of LPS in Gram-negative, and teichoic acids in Gram-positive, bacteria. In contrast to that, mammalian membranes predominantly consist of zwitterionic phospholipids. Likewise, the presence of cholesterol reduces the activity of AMPs towards membranes [7,9-12].

The predominant human AMPs are histatins, defensins and a single, 37-amino acid long (4.5 kDa) cathelicidin named LL-37 [13]. While histatins are a class of AMPs found in saliva and are thus just indirectly involved in lung homeostasis [13], several defensins and cathelicidin LL-37 play an essential role in lung innate immunity [14]. Actually, $\alpha$-defensins are highly present in neutrophils, $\beta$-defensins mainly in airway epithelia cells and LL-37 in neutrophils and airway epithelia cells [6,15]. Indeed, LL-37 seems to be the most prominent AMP in bronchoalveolar fluid, with a concentration of up to $25 \mu \mathrm{g} / \mathrm{mL}$ [16], and several studies revealed that LL-37 is clearly involved in defense of the lung against bacterial infections [14]. The best-known effect of LL-37 on bacteria is the perforation of their membranes via toroidal pore formation [7,17-19]. Furthermore, LL-37 interrupts the bacterial cell wall synthesis and potentially interacts with pathogenic intracellular protein targets like chaperones [10,20]. Moreover, the disturbance of peptidoglycan synthesis [7] and neutralization of bacterial toxins other than Gram-negative-derived LPS [10] seem plausible. Finally, LL-37 can act as a chemokine to attract leukocytes to the side of infection. This process initiates a diverse immune response against pathogens and elevates the local LL-37 concentration to an even higher level [21].

Considering these modes of action, the antimicrobial peptide LL-37 represents an important stress factor for bacteria, and therefore molecular effectors evolved that reduce the damaging impact of the AMP on the bacterial physiology [9]. Known adaptation mechanisms include the repulsion of LL-37 by cell-surface adaptations, interception or the targeted binding of LL-37 by the release or reconstruction of surface molecules, the export of LL-37 by ABC-transporters and the degradation or modification of LL-37 [7,22]. The repulsion of LL-37 by cell surface adaptations inhibits the initial interaction between the peptide and the bacterial membrane or cell wall. This is mainly accomplished by modifications to the surface charge. The reduction in negative charges or the introduction of positive charges to bacterial membranes and associated structures like teichoic acids and peptidoglycan decreases the attraction of cationic LL-37 [23-25]. The interception or targeted binding of LL-37 is thought to be mediated by the release of surface molecules like capsular polysaccharides, teichoic acids and peptidoglycan or the possible restructuring of the peptidoglycan scaffold [16,26,27]. Potentially, the effects of intracellular LL-37 can be counteracted by specific bacterial efflux pumps exporting these peptides [28,29]. In fact, S. pneumoniae expresses more than 300 transporters. For a visual overview see: http://www.membranetransport.org/transportDB2/index.html [30]. Lastly, the degradation of LL-37 by proteases, or the hypothetical charge modification of the AMP, would decrease the interaction with the bacterial cell surface. Indeed, this represents a common strategy, that is potentially involved in LL-37 resistance [31,32].

Although adaptations to LL-37 are already described for some bacteria, it remains to be elucidated how S. pneumoniae, the leading cause of bacterial pneumonia, reacts to LL-37 stress on a functional level. In this study, gel-free liquid chromatography-mass spectrometry (LC-MS)-based proteomics was used to analyze the proteome of pneumococci under LL-37 stress compared to untreated pneumococci for the first time.

As carriage of S. pneumoniae is a prerequisite for infection [3], understanding the interaction between innate immune molecules, specifically AMPs, and pneumococci would give us more opportunities to fight this widespread pathogen. In fact, enhancing the effect of existing antibiotics via the application of antimicrobial peptides was shown and could be an interim solution to fight drug-resistant strains. 
Presumably, the membrane disruption effect of AMPs promotes access for classical antibiotics into bacterial cells, and this would also explain the synergy between AMPs and antibiotics [7,13,33]. Moreover, resistance against classical antibiotics sensitizes bacteria for antimicrobial peptides [34]. On the other hand, attempts to block the bacterial resistance system to already existing AMPs in the human body continue to be a promising medical approach [23].

\section{Materials and Methods}

For the present study, the following workflow was established by the optimization of numerous parameters including cultivation, protein extraction, digestion, peptide fractionation, LC-MS/MS methods and database search (Figure 1).

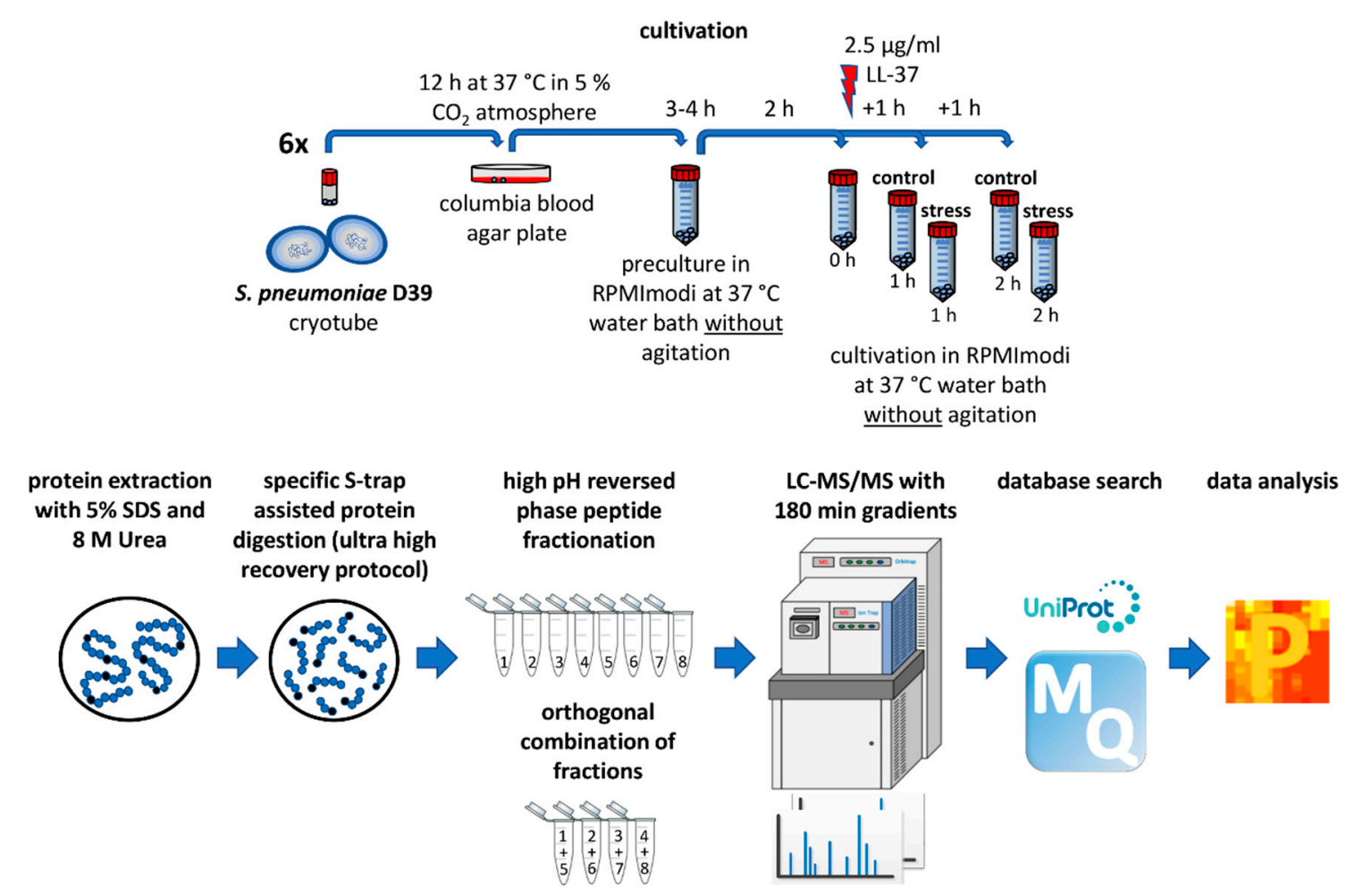

Figure 1. Established workflow for proteomic investigation of LL-37 stress on pneumococci.

\subsection{Bacteria and Cultivation}

The extensively characterized Streptococcus pneumoniae D39 strain (serotype 2, NCTC 7466) was taken from cryo culture and incubated for $12 \mathrm{~h}$ on Columbia blood agar plates at $37{ }^{\circ} \mathrm{C}$ under $5 \% \mathrm{CO}_{2}$ atmosphere. Bacteria were then transferred for pre-cultivation to $40 \mathrm{~mL}$ modified RPMI 1640 media (Thermo Fisher Scientific, Rockford, IL, USA) (further named chemically defined media = CDM) [35] and the bacterial growth was photometrically observed while cultivating at $37{ }^{\circ} \mathrm{C}$ in a water bath without agitation. When the culture reached an optical density $\left(\mathrm{OD}_{600}\right)$ of 0.5 , cells were diluted in $40 \mathrm{~mL}$ of $\mathrm{CDM}$ to a starting $\mathrm{OD}_{600}$ of 0.07 to receive the main culture. Cultivation was performed under the same parameters as before. After $2 \mathrm{~h}$ of bacterial growth, $2.5 \mu \mathrm{g} / \mathrm{mL}$ LL-37 acetate (Innovagen, Lund, Sweden) was added to the stress samples. For controls, an equivalent volume of distilled water $(100 \mu \mathrm{L})$ was added. Finally, pneumococci were harvested by centrifugation $\left(25 \mathrm{~min}, 10,000 \times g, 4{ }^{\circ} \mathrm{C}\right.$, soft deceleration) directly before the addition of stress and after 1 and $2 \mathrm{~h}$ of LL-37 treatment together with the corresponding control samples. Bacterial pellets were frozen at $-80^{\circ} \mathrm{C}$ until further processing. Experiments were performed in six independent biological replicates. 


\subsection{Colony Forming Unit (CFU) Assay}

Bacteria were grown, stressed and harvested as described before (Section 2.1) in three biological replicates. Following that, cell pellets were diluted to an $\mathrm{OD}_{600}$ of 1 in $1 \mathrm{~mL}$ sterile phosphate-buffered saline (PBS) [36] corresponding to about $10^{9}$ bacteria. A total of $100 \mu \mathrm{L}$ of further $(1: 100,000)$ diluted samples were pipetted on blood agar plates. The plates were homogenously covered with bacterial suspension using glass pearls. After the removal of pearls, the plates were incubated for $11 \mathrm{~h}$ at $37^{\circ} \mathrm{C}$ under $5 \% \mathrm{CO}_{2}$ atmosphere and the colonies were counted.

\subsection{LL-37 Sensitivity Testing}

S. pneumoniae $\mathrm{D} 39$ wildtype (wt), as well as $\Delta d l t D$ and $\Delta l i c D 2$ deletion mutants, were cultivated and stressed as mentioned before (Section 2.1). For evaluating the sensitivity of pneumococci towards LL-37, levels of reduction in optical density caused by the peptide were measured in triplicate experiments in the three strains studied. Differences between mutant and wt inhibition were statistically analyzed using one-tailed $t$-tests assuming unequal variance, $p<0.05$.

\subsection{Mutant Construction}

The pneumococcal licD2 mutant in S. pneumoniae D39 was constructed by amplification of a DNA fragment consisting of the S. pneumoniae D39 licD2 gene and $~ 500$ bp up and downstream flanking regions using polymerase chain reaction (PCR) with primer LicD2SphIfor (5'-GCGCGCGCAT GCTATTGATA CTCGAAATAC AAAAACCTAT G-3') and LicD2SacIrev (5'-GCGCGCGAGC TCTGGTAAGA TTGGTGATGA CGATAAGG-3'). The purified PCR product was cloned into plasmid pUC18 (Thermo Fisher Scientific, Rockford, IL, USA). The resulting plasmid was used as a template for an inverse PCR using primer Invrev1130BamHI (5'-GCGCGCGGAT CCCCTAATCC TCCAATTTAT AAGCG-3') and Invfor1130SmaI (5'-GCGCGCCCCG GGTTGAGGGG GATTATACAA ACTAC-3'). Afterwards, an ermB gene, amplified by PCR from vector pTP1 [37] using primer InvrevBamHIErm (5'-GCGCGCGGAT CCACGGTTCG TGTTCGTGCT GACTTGC-3') and InforSmaIErm (5'-GCGCGCCCCG GGGTAGGCGC TAGGGACCTC TTTAGC-3') was inserted. The final recombinant plasmid was used to transform and mutagenize S. pneumoniae D39.

For the construction of $S$. pneumoniae D39 $\Delta d l t D$, a DNA fragment containing the dltD gene and $\sim 500$ bp up and downstream of dltD was amplified by PCR using Primer DltDforXbaI (5'-GCTCTAGACG AGTGGTCCAA TCGATCGCTT TAAGC-3') and DltdrevSacI (5'-GCGAGCTCCC AAGCGTTTGT CTCGATGTTC CCACATG-3'). The purified PCR fragment was cloned into the pMiniT vector (NEB) and the resulting vector was used as a template for an inverse PCR with primer InvKpnI (5'-GCGCTCTAGA GCGTTTAAGC ATTTTGTAGC TC-3') and InvNcoI (5'-GCGCCCATGG GATGGAGATG TCAAAGAATT TCAAT-3'). The ermB gene, amplified from plasmid pTP1 [37] using primer ermforw (5'-GATGATGATG ATCCCGGGTA CCAAGCTTGA ATTCACGGTT CGTGTTCGTG CTG-3') and ermrevNcoI (5'-GCGCCCATGG CGTAGGCGCT AGGGACCTC-3') was cloned into the inverse PCR product. The final vector was used to transform and mutagenize S. pneumoniae D39.

\subsection{Sample Preparation for LC-MS Measurements}

\subsubsection{Protein Extraction and Determination}

Loss of hydrophobic proteins during protein extraction was minimized by resuspension of pneumococcal pellets in $1 \mathrm{~mL} 50 \mathrm{mM}$ triethylammonium bicarbonate (TEAB) lysis buffer ( $\mathrm{pH} 7.55$ ) containing $5 \% \mathrm{SDS}(\mathrm{wt} / \mathrm{wt}$ ) and $8 \mathrm{M}$ urea. Subsequently, the bacterial suspension was pipetted to cyro tubes filled with $500 \mu \mathrm{L}$ glass beads (diameter: $0.10-0.11 \mathrm{~mm}$ ). Cells were disrupted by bead beating $(6 \mathrm{~m} / \mathrm{s}, 4 \times 30 \mathrm{~s}$ with $120 \mathrm{~s}$ breaks on ice) and subsequent sonication $(1 \times 60 \mathrm{~s})$. In order to separate the protein-containing supernatant from cell debris, a centrifugation step was performed $(8 \mathrm{~min}, 13,000 \times g$, $\left.4{ }^{\circ} \mathrm{C}\right)$. 
Protein content was determined using BCA-assays (Pierce BCA Protein Assay Kit; Thermo Fisher Scientific, Rockford, IL, USA) following the manufacturers protocol.

\subsubsection{Protein Digestion}

For tryptic digestion of $50 \mu \mathrm{g}$ protein, S-trap spin columns (Protifi, Huntington, NY, USA) were used in combination with the manufactures "ultra-high recovery protocol" available under https://www.protifi.com/resources. Shortly, protein samples were reduced and alkylated and loaded into the suspension traps. Then, the protein solution was acidified, and trypsin added (Promega, Madison, WI, USA) and immediately washed several times. After the addition of further trypsin to the matrix, the trap was incubated for $1 \mathrm{~h}$ at $47^{\circ} \mathrm{C}$, enabling protein digestion. Lastly, peptides were eluted with acetonitrile and dried using a vacuum concentrator.

\subsubsection{Peptide Fractionation}

To increase the proteomic coverage, peptides were fractionated following the basic $\mathrm{pH}$ reversed-phase peptide fractionation protocol (Thermo Fisher Scientific, Rockford, IL, USA) using self-packed columns filled with $18 \mu \mathrm{g}$ of Reprosil-Gold 300 C18, $5 \mu \mathrm{m}$ material (Dr. Maisch, Ammerbuch-Entringen, Germany). Basically, peptides were loaded into the column, washed and eluted into eight different fractions using increasing concentrations of acetonitrile in a high-pH solution ( $0.1 \%$ triethylamine). Afterwards, the fractions $1 \& 5,2 \& 6,3 \& 7$ and $4 \& 8$ were orthogonally combined for optimal use of LC-MS analysis time according to the principles described elsewhere [38].

Finally, peptides were dried and suspended in $40 \mu \mathrm{L} 0.5 \times$ iRTs in water with $0.1 \%$ acetic acid (Biognosys, Schlieren, Switzerland) for LC-MS quality control purposes.

\subsection{LC-MS Measurements}

Samples were measured on an LTQ Orbitrap Velos mass spectrometer in combination with an EASY nLC-1000 liquid chromatography system. First, $2 \mu \mathrm{L}$ of the sample was loaded with $12 \mu \mathrm{L}$ buffer A (water with $0.1 \%$ acetic acid) on an in-house packed C18 column (ReproSil-Pur 120 C18-AQ, $3 \mu \mathrm{m}$ material) of $20 \mathrm{~cm}$ length and $100 \mu \mathrm{m}$ inner diameter. Then, a non-linear 180-min gradient from $1 \%$ to $99 \%$ buffer B (acetonitrile with $0.1 \%$ acetic acid) was used for the elution of peptides with a flowrate of $300 \mathrm{nl} / \mathrm{min}$. The MS measurement was performed in data-dependent mode. The MS1 scans covered a mass range of 300-1700 m/z at a resolution of 30,000 (at $400 \mathrm{~m} / \mathrm{z}$ ) and the 20 most intense precursors were selected and fragmented via collision-induced dissociation (CID) using a normalized collision energy of 35. Ions with unknown charge or charge one were excluded from fragmentation. Fragment ions were measured in the ion trap (MS2). Additionally, precursors were excluded for $20 \mathrm{~s}$ after fragmentation for additional fragmentation.

\subsection{Quality Control and Data Analysis}

\subsubsection{Quality Control}

Data quality was monitored by adding synthetic iRT peptides (Biognosys, Schlieren, Switzerland) to the samples, thereby checking for potential shifts in mass deviation or retention time. Additionally, MaxQuant output files and RawMeat (VAST Scientific, Cambridge, MA, USA) were used to detect unexpected changes in intensity, the number of obtained scans, the distribution of TopN or identification rates. Finally, hierarchical clustering and a principle component analysis confirmed the quality of our data, showing clustering of replicates, sample points and conditions (Figure S1). Only data passing the quality checks were used in this study.

\subsubsection{Protein Identification and Quantification}

MaxQuant version 1.6.5.0 and its Andromeda search engine [39] was used for protein identification and quantification with the following settings. Trypsin/P was selected as a protease that cleaves after 
lysine and arginine independent of following prolines. The maximal number of missed cleavages was set to 2. Likewise, the minimal number of unique peptides per protein group was set to 2 to be considered as identified. Oxidation (M) was considered as a variable and carbamidomethyl (C) as a fixed modification, whereas the maximal number of modifications per peptide was 5. For quantification, solely unique peptides were used. Databases loaded to MaxQuant were: the S. pneumoniae D39 proteome containing 1915 proteins obtained from Uniprot.org (Proteome ID: UP000001452, last modified November 9, 2018), the MaxQuant contaminants file and sequences for human LL-37, yeast enolase and iRT peptides.

\subsubsection{Data Evaluation}

Data were analyzed using Perseus [40] version 1.6.5.0. Initially, the quantitative values were transformed using the function: $\log _{2}(\mathrm{x})$. Next, values were filtered based on the criteria: only identified by site, reverse, potential contamination, identified in at least $5 / 6$ biological replicates under at least $1 / 5$ condition. Missing values were imputed from normal distribution using the Perseus standard settings. For examination of differences between stress and control samples, Student's $t$-tests were used (S0: 0, side: both, use for truncation: $p$-value, threshold $p$-value: 0.01$)$. Furthermore, a minimum fold change of 1.5 was defined to extract the most prominent proteomic differences between the tested conditions, which are expected to have an impact on physiology. Lastly, protein annotations were made using the S. pneumoniae D39 proteome obtained from Uniprot.org (Proteome ID: UP000001452, last modified November 9, 2018) supplemented with data obtained from PneumoBrowse [41], the subcellular localization database PSORTdb 3.0 [42] and information on the two-component regulatory system CiaHR [43].

\subsection{Data Availability}

The mass spectrometry proteomics data have been deposited to the ProteomeXchange Consortium (http://proteomecentral.proteomexchange.org) via the PRIDE [44] partner repository with the dataset identifier PXD016511.

\section{Results}

To investigate the proteomic response of pneumococci towards LL-37, S. pneumoniae D39 was stressed by the addition of $2.5 \mu \mathrm{g} / \mathrm{mL}$ LL-37 during the exponential growth phase (two hours after the inoculation of main cultures) that was expected to be physiologically relevant. Furthermore, pre-experiments showed a clear and concentration-dependent antibacterial effect of the peptide, ranging from a profound inhibition of bacterial growth using $20 \mu \mathrm{g} / \mathrm{mL}$ of LL-37 to almost no inhibition by adding $0.625 \mu \mathrm{g} / \mathrm{mL}$ peptide to pneumococcal cultures.

In our experiment, the application of stress reduced the maximal optical density $\left(\mathrm{OD}_{600}\right)$ of the cultures by about $20 \%$ (Figure 2A). Moreover, the viability of stressed cells was confirmed via colony-forming unit (CFU) assays (Figure 2B). Five samples, $1 \times$ before the addition of LL-37, $2 \times$ after $1 \mathrm{~h}$ of stress (control, stress) and $2 \times$ after $2 \mathrm{~h}$ of stress (control, stress), were harvested in six biological replicates and prepared for MS, as shown in Figure 1. This allowed us to link the significant reduction in optical density of the stressed bacterial cultures to their proteomic state. 

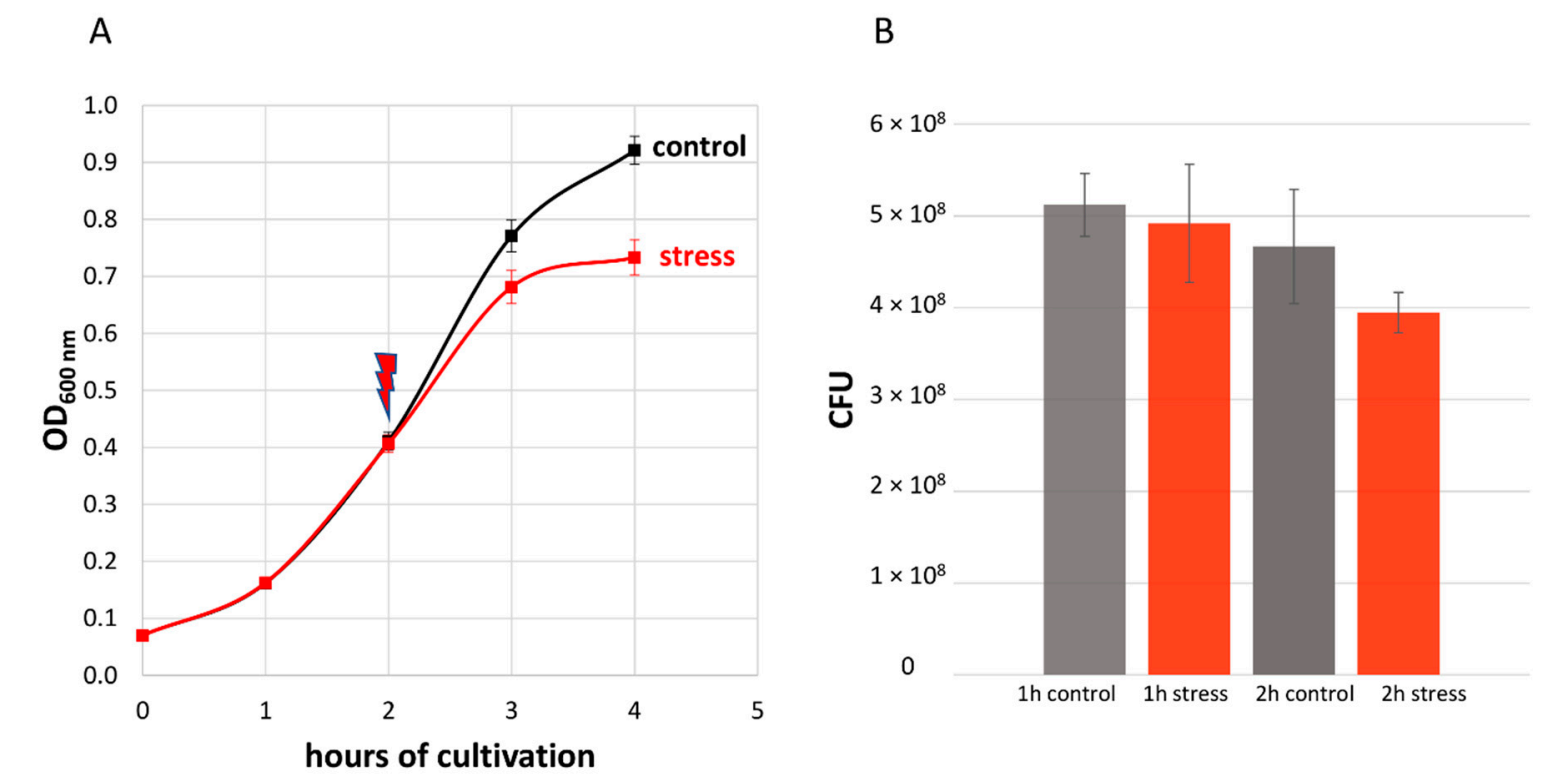

Figure 2. (A) Effect of $2.5 \mu \mathrm{g} / \mathrm{mL}$ LL-37 on the growth of S. pneumoniae D39. The red arrow indicates the application of the stress after two hours of cultivation. Error bars represent the standard deviation. $n=6$. (B) Colony-forming units (CFU) per $\mathrm{ml} \mathrm{OD}_{600}=1$ bacterial suspension. Samples were taken every hour after the addition of $2.5 \mu \mathrm{g} / \mathrm{mL}$ LL-37. Means are shown and error bars represent the standard deviation. $n=3$.

In total, $68 \%$ of the pneumococcal proteome (1293/1915 proteins) could be identified. Proteins annotated to be cytoplasmic or cell-wall-bound could be identified as almost $80 \%$. Additionally, membrane proteins, extracellular proteins and proteins of unknown localization were covered to about $50 \%$. Finally, the quantitative correlation between the data of biological replicates was at least $97.7 \%$ (Figure 3, Figures S2-S6), demonstrating the high reproducibility of our experimental approach.

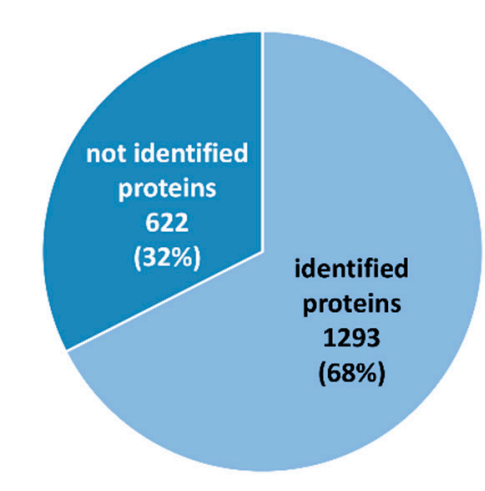

Proteome coverage Total annotated proteome: 1915

\begin{tabular}{|l|l|l|}
\hline bacterial fraction & $\begin{array}{l}\text { experimental number of proteins/ } \\
\text { theoretical number of proteins }\end{array}$ & $\%$ \\
\hline cytoplasmic & $810 / 1028$ & 79 \\
\hline $\begin{array}{l}\text { cytoplasmic } \\
\text { membrane }\end{array}$ & $303 / 529$ & 57 \\
\hline unknown & $142 / 296$ & 48 \\
\hline extracellular & $18 / 36$ & 50 \\
\hline cell wall & $20 / 26$ & 77 \\
\hline total proteome & $1293 / 1915$ & 68 \\
\hline
\end{tabular}

Pearson correlation of 6 replicates

$0 \mathrm{~h}: \min .0 .977$

$1 \mathrm{~h}$ control: $\min .0 .985 \quad 1 \mathrm{~h}$ stress: $\min .0 .986$

2 h control: $\min .0 .982 \quad 2$ h stress: $\min .0 .986$

Figure 3. Graphical representation of proteome coverage and correlation of replicates.

As the obtained dataset passed all executed quality control steps, Student's $t$-tests were used for the evaluation of differences in the proteome of stressed and unstressed pneumococci consisting of 1118 proteins after value-filtering in Perseus. One hour after application of AMP stress, 45 proteins were significantly changed in abundance (33 less abundant, 12 higher abundant) and $2 \mathrm{~h}$ after stress 78 proteins ( 38 less abundant, 40 higher abundant) fulfilled the strict $t$-test's criteria to be considered as changed (Figure 4). Furthermore, many proteins that were changed after $1 \mathrm{~h}$ of stress were also found to be significantly changed in abundance after $2 \mathrm{~h}$ hours of stress in a similar manner. 
$1 \mathrm{~h}$ after stress

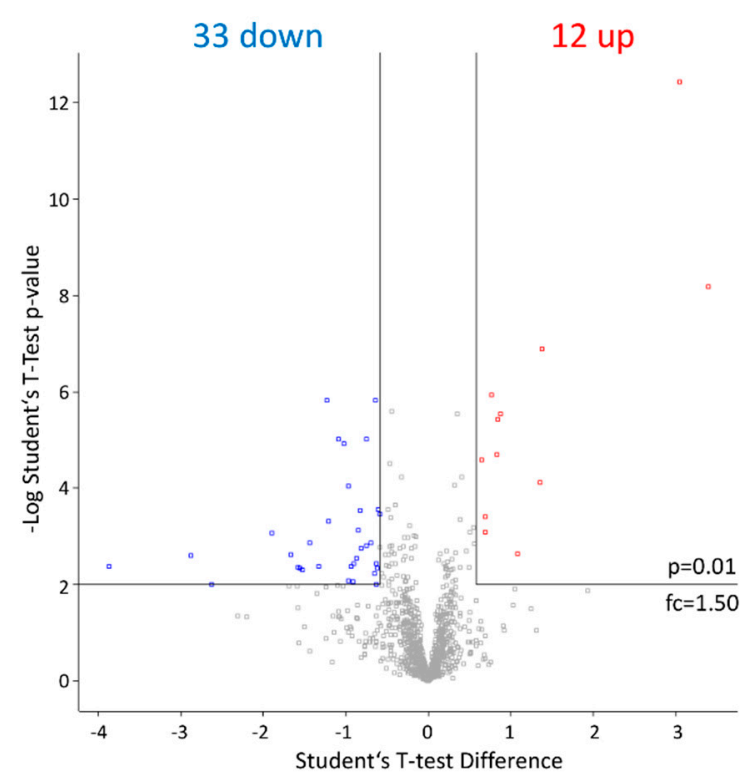

$2 \mathrm{~h}$ after stress

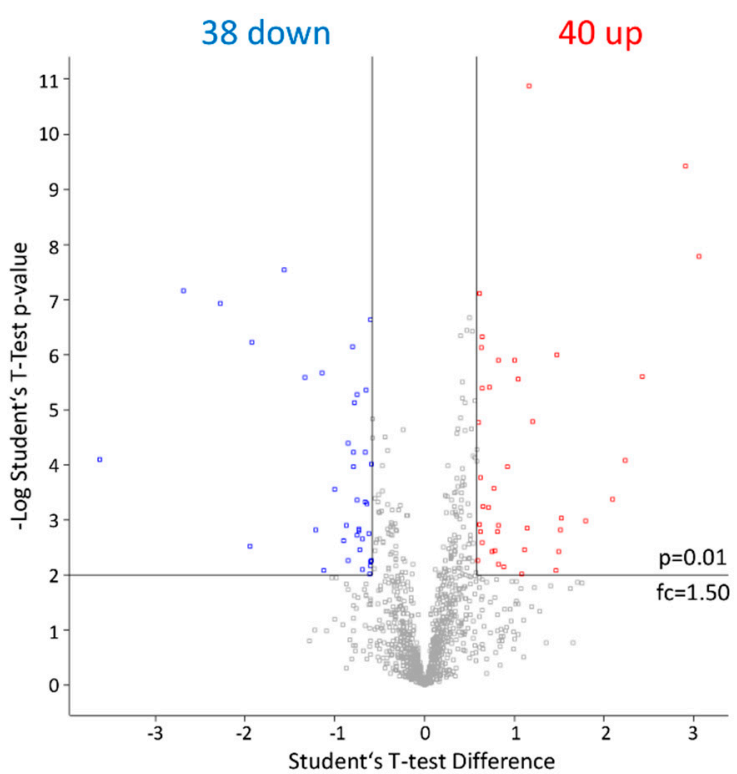

Figure 4. Graphical representation of significantly\# affected proteins after LL-37 treatment via Volcano plots. Proteins with an increase in abundance after application of the stress are labeled in red and proteins with a decrease in protein abundance in blue, respectively. \# Student's $t$-test ( $p$-value $=0.01$, min. fold change $=1.5$ ).

Subsequently, significantly changed proteins could be classified into functional groups: transporters (29 proteins), proteins involved in gene regulation (11), a single protease (1), metabolic proteins (12), cell surface modification proteins (7), virulence factors (7) and uncategorized proteins (other (27) and unknown (11)). Hence, a total number of 105 proteins with a significantly changed amount (43 higher in abundance, 62 lower abundant) could be identified after the addition of LL-37 (Figure 5, Table S1).

\section{5 significantly changed proteins}

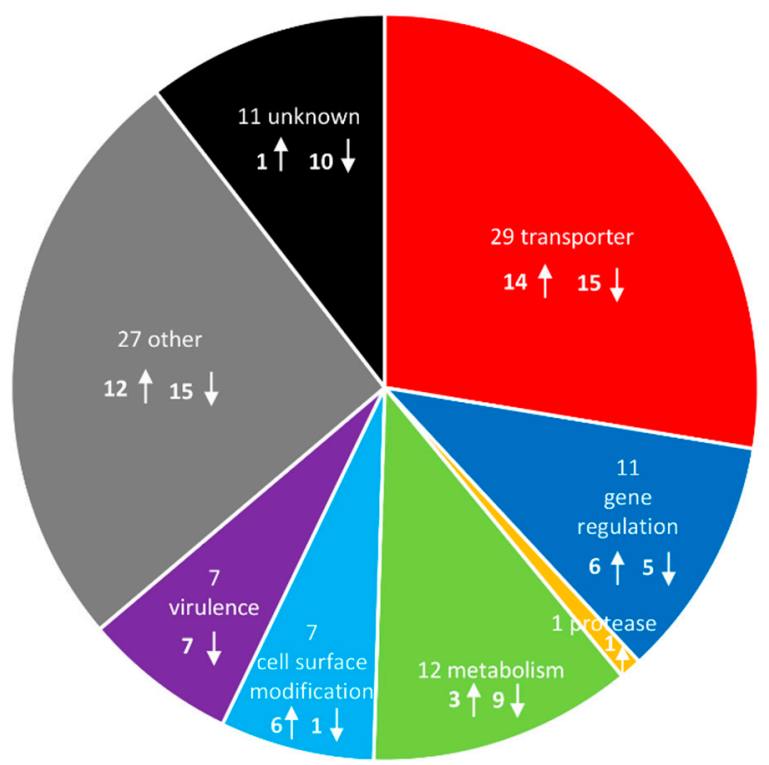

Figure 5. Graphical representation of significantly\# affected proteins and their function after LL-37 treatment. The arrows indicate an increase or a decrease in protein abundance after application of the stress, respectively. \# Student's $t$-test ( $p$-value $=0.01$, min. fold change $=1.5$ ). 
Before in-depth analyses, the proteome data were compared to publicly available high-quality genomics and gene regulation data for the pneumococcus $[41,43]$ and a transcriptomics dataset for pneumococci after LL-37 stress [45]. Briefly, proteins were considered for further analyzation if at least two criteria were fulfilled. These include bacterial resistance candidates with significantly changed abundances on proteome and transcriptome levels, proteins that were found to be changed at both timepoints on proteome level (after $1 \mathrm{~h}$ and $2 \mathrm{~h}$ of stress), hits of several proteins of a single operon, or if levels of a protein and its corresponding regulator were significantly altered after LL-37 stress application. This resulted in a new, extensively examined dataset (Figure 6, Table S2).

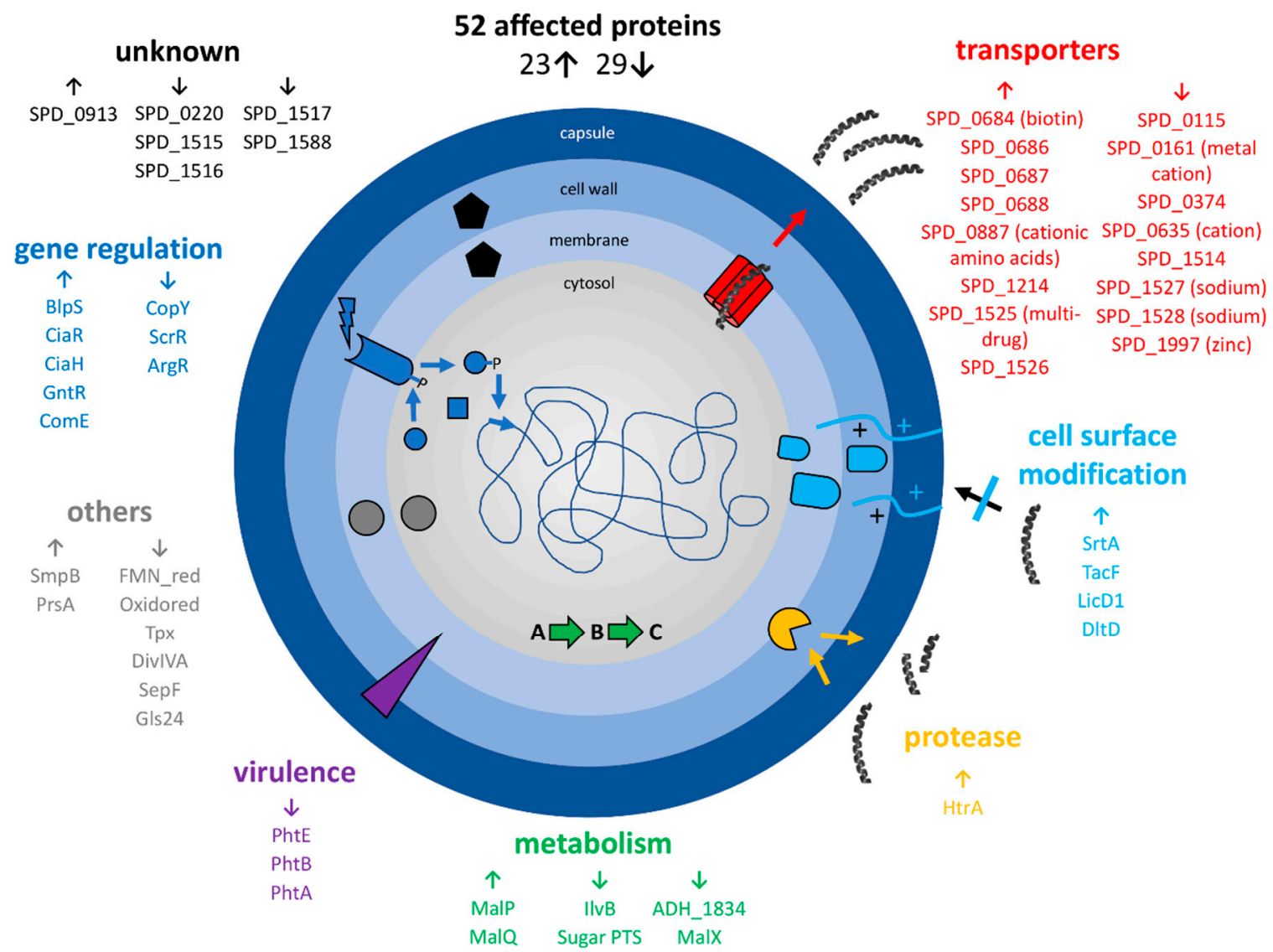

Figure 6. Summary of selected proteomic changes of S. pneumoniae D39 upon LL-37 exposure after comparison of significant\# proteome changes with published transcriptome and genome data. The arrows indicate an increase or a decrease in protein abundance after application of the stress, respectively. \# Student's $t$-test ( $p$-value $=0.01$, min. fold change $=1.5$ ).

Basically, the abundance of many transporters, especially ABC-transporters, changed significantly and often drastically after LL-37 exposure. Eight proteins annotated as transporters were increased in abundance and eight proteins of this class decreased. Most prominent was the more than 8-fold increase in the multidrug ABC transporter SPD_1525-26 after 2h of stress (7.5-fold after $1 \mathrm{~h}$ of stress) and the significant decrease in SPD_1527-28 sodium ABC exporter (down to 0.4-fold after 1h of stress and 0.3-fold after $2 \mathrm{~h}$ of stress for SPD_1527). Likewise, the transcriptional regulator SPD_1524 (GntR) was found in considerably higher abundance after LL-37 stress. Additionally, the sensor histidine kinase $\mathrm{CiaH}$ and the response regulator $\mathrm{CiaR}$ increased after stress at both timepoints. Other proteins involved in gene regulation with a moderately higher level after LL-37 treatment are BlpS (fold change (fc) of 1.8 at the second timepoint) and ComE (fc of 1.5 at the same sample point). Besides that, the heat-inducible serine protease and chaperone HtrA was highly enriched in stress samples (fc of 2.6 and 2.0, 1h and $2 \mathrm{~h}$ after stress, respectively) and the bacterial metabolism was clearly affected by LL-37, illustrated 
especially by higher amounts of MalP, MalQ and lower amounts of MalX. While proteins involved in cell surface modification (DltD, LicD1, TacF, SrtA) showed significantly higher abundance under stress, particularly after $2 \mathrm{~h}$ of stress, several virulence factors with a role in adhesion (histidine triad proteins A, B and E) were less abundant upon stress with LL-37. Furthermore, proteins involved in correct protein-folding (foldase PrsA) and biosynthesis (SsrA-binding protein SmpB) were more abundant, and proteins involved in cell redox homeostasis (Tpx) and cell division (SepF, DivIVA) were less abundant after LL-37 exposure. Lastly, the unknown proteins SPD_0913 (accumulated up to a fc of 2.8) and SPD_1515-17 (decreased clearly after $2 \mathrm{~h}$ of stress) changed significantly.

Based on the significant changes seen in the proteomic data, two mutants were chosen and subjected to LL-37 sensitivity testing to support the putative role of the corresponding proteins in LL-37 adaptation (Figure 7). This was done by stressing pneumococci and comparing the reduced optical density of the stressed cells with the ones from the control condition. First, the sensitivity of the S. pneumoniae D39 $\Delta d l t D$ mutant towards LL-37 was evaluated, as DltD is known to effect resistance to AMPs in pneumococci [46] and was also found to be more abundant in our dataset after LL-37 treatment. Additionally, S. pneumoniae D39 $\Delta$ licD2 was selected because LicD2 showed a similar change in abundance after LL-37 stress to the significantly increased genomic neighbor LicD1 (without passing the strict statistical parameters). Moreover, a deficiency in licD1 appears to be lethal [47]. As shown in Figure 7 , the growth of the wildtype D39 strain is reduced by about $21 \%$ after peptide stress compared to the control condition, whereas growth of $\Delta d l t D$ and $\Delta l i c D 2$ mutants are significantly reduced by about $28 \%$ and $29 \%$ in comparison to the untreated strains, respectively.

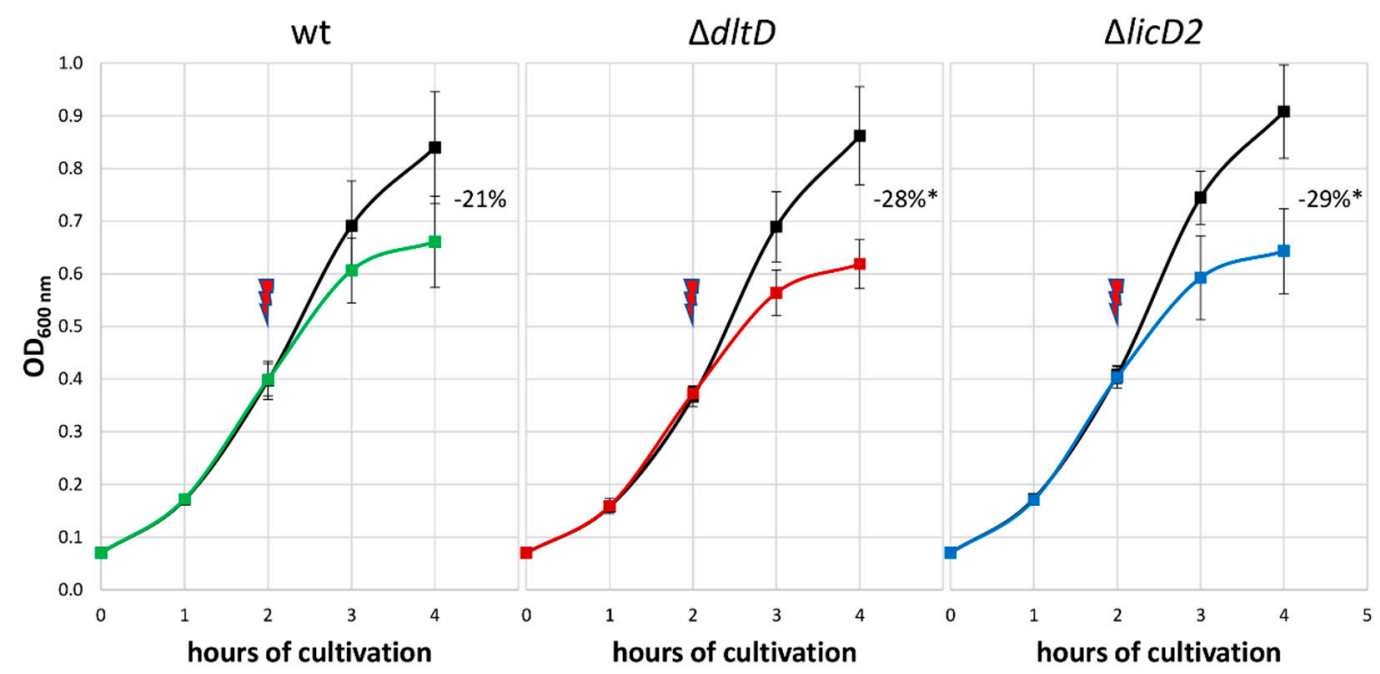

Figure 7. Effect of $2.5 \mu \mathrm{g} / \mathrm{mL}$ LL-37 on the growth of S. pneumoniae D39 wildtype (wt) in comparison to stressed D39 $\Delta d l t D$ and D39 $l i c D 2$ mutants. The red arrow indicates the application of the stress after two hours of cultivation. The numbers represent the growth inhibition of stressed pneumococci compared to corresponding untreated D39 strains in percentage. ${ }^{*}$ Significant difference between mutant and wt inhibition using one-tailed t-tests assuming unequal variance, $p<0.05$. The error bars represent the standard deviation. $n=3$.

\section{Discussion}

The presented dataset shows the highest proteome coverage for the S. pneumoniae D39 wildtype known to us. Furthermore, 105 proteins could be identified as significantly changed after LL-37 stress, while stressed bacteria showed a similar but about $20 \%$ reduced growth compared to unstressed cells. A total of 52 protein candidates with a potential specific function in LL-37 adaptation could be identified after comparison with transcriptomic and genomic data.

Among these candidates are the teichoic acid flippase TacF (SPD_1128) [48] and LicD1 (SPD_1129), an enzyme responsible for transfer of phosphorylcholine to teichoic acids, thereby mimicking eukaryotic 
membranes, increasing the surface charge and adding binding sites for choline binding proteins to the cell wall [24,49]. Interestingly, both corresponding RNA levels [45] and the protein abundance after $1 \mathrm{~h}$ of stress are not significantly affected, but TacF and LicD1 proteins are significantly more abundant after $2 \mathrm{~h}$ of stress, suggesting a relatively slow regulation of protein level. Additionally, DltD (SPD_2002), which alanylates teichoic acids and hence introduces positive charges to the cell surface [46], was more abundant in stressed bacteria (after 1 and $2 \mathrm{~h}$ ) in accordance with the accumulation of its RNA in the transcriptomic dataset [45]. Next, the enzyme sortase SrtA is more abundant under 1 and $2 \mathrm{~h}$ of stress, attaching LPXTG-motif proteins to the cell surface. Taking all that into account, it seems plausible that pneumococci are also able to repel the positively charged LL-37 by cell wall adaptations. In fact, a reduction in negative surface charge would decrease the electrostatical forces between the cationic peptide (charge +6$)$ and the bacteria, and thus decrease its antimicrobial effects. This appears even more likely when considering that involvement of the dlt- and lic-operons in resistance to LL-37 in other bacteria or of S. pneumoniae against other cationic antimicrobial peptides was already described. In fact, non-functional $d l t$ affected the resistance of $S$. pneumoniae to the bacterial secreted lantibiotics nisin and gallidermin [46], and a lic mutation the susceptibility of Haemophilus influenzae towards LL-37 [24]. Alternatively, LTA release, aiming for targeted binding of LL-37, would be an effective interception mechanism against the action of the peptide [27], but was not examined in this study.

Furthermore, the abundance of several transporters, especially of the ABC type, was changed in response to LL-37. Consistently using the annotation from TransportDB 2.0, the proteins with transporter function appear to be enriched among the significantly changed proteins ( 23 out of 105; $22 \%$ ), as there are 304 transporters annotated for the pneumococcal proteome (16\%). The same trend can be observed comparing the number of significantly changed transporters with the transporters identified in our experiment (194 among 1293; 15\%). These changes could lead to the removal of LL-37 from the bacterial membrane, as shown for other bacteria and other cationic antimicrobial peptides [50-52]. Interestingly, the accumulated transporter proteins are mainly regulated by GntR (SPD_0686-88, SPD_1525-26) and the depleted ones by ComE (SPD_0374, SPD_1527-28), regulators that are both more abundant after the addition of LL-37. The observation that many of the transporters increased after stress, including the ones independent of GntR, has also been made on the RNA level [45].

Besides that, the serine protease and chaperone HtrA (SPD_2068) is highly enriched in stress samples. This finding could be explained by a potential LL-37-degrading function of the enzyme, an enhanced removal of damaged or misfolded proteins in response to cell wall stress, or the role of $\mathrm{HtrA}$ in competence (ComC degradation) and bacteriocin (BlpC degradation) regulation [53].

Then, the amount of proteins encoded by the maltose operon (MalP, MalQ) probably changed due to changes in CiaR amounts. However, how this observation is connected to AMP adaptation is still unclear.

Interestingly, three surface virulence proteins, the histidine triad protein A, B and E, are depleted during stress, supposedly to reduce interaction with the immune system [54]. As the virulence proteins are regulated by the $\mathrm{Zn}^{2+}$-dependent regulator $\mathrm{AdcR}$, it was unexpected that AdcR did not change significantly. However, posttranslational modifications or ligand-binding could alter the regulator's activity. Probably for the same reason, LytA was not found to be enriched even when the involvement of this enzyme in LL-37 resistance was described for pneumococci [16]. In fact, LytA activation is regulated by phosphorylation [55], and this could also be the case in this study.

Other proteins found to be regulated are foldase PrsA (accumulated at both sample points but expression was not significantly affected) and several proteins involved in cell redox homeostasis, e.g., the thioredoxin peroxidase Tpx (SPD_1464) (shows the same trend on RNA level [45]), and cell division (SepF, DivIVA) (all three proteins are depleted). As foldases are chaperones and support correct protein folding, presumably there is an enhanced accumulation of misfolded proteins after LL-37 stress in pneumococci. Furthermore, virulence factors seem to be reduced after LL-37 stress 
(depletion of histidine triad proteins) and a hypothetical decreased production rate of host-damaging $\mathrm{H}_{2} \mathrm{O}_{2}$ would explain the reduced synthesis of redox homeostasis proteins in pneumococci.

On the other hand, the regulation of pneumococcal adaptation in response to LL-37 seems to be mainly mediated by the transcriptional regulator GntR (SPD_1524) and the two-component systems, TCS05 CiaHR, TCS12 ComDE and TCS13 BlpHR (BlpS). In detail, GntR shows a substantial fold change at both timepoints, and corresponding RNA was also found to be considerably more abundant [45]. Next, both components of the CiaHR system, the sensor histidine kinase CiaH and the response regulator CiaR, are more abundant under stress on proteome and transcriptome level [45]. Finally, the response regulator part of ComDE (ComE) was increased on the protein level and the BlpHR two-component system was indirectly affected by an increase in BlpS protein and RNA in pneumococci after LL-37 stress. Indeed, this makes sense considering the complex interaction of Cia, Com and Blp regulatory systems [53], including the CiaHR-regulated degradation in ComC and BlpC signaling peptides by HtrA. After focusing on targets of the CiaHR regulon (probably activated by surface stress) [53] it becomes clear that it shows many of the effects known to be induced by its activity [43], among them, the accumulation of serine protease HtrA, foldase PrsA, the metabolic proteins MalP and MalQ and the unknown function protein SPD_0913.

Lastly, the data indicate that the unknown proteins SPD_0913, that was similarly induced on RNA level [45], and SPD_1515-17 (identified as less abundant uniquely on proteome level), play a potential role in innate immunity resistance. Perhaps SPD_1515-17 sensitizes pneumococci toward LL-37, and therefore a decreased abundance of this protein, and other proteins reduced in abundance after LL-37 stress, would result in a higher tolerance to the peptide. Backing up this hypothesis, SPD_1515-16 are annotated as membrane proteins, being potentially in contact with membrane-acting LL-37. However, the structures of these proteins are unknown, so that no statement can be made regarding a potential negative surface charge of this protein that would attract cationic AMPs.

For the two proteins DltD and LicD2, the genomic neighbor of significantly regulated LicD1, the role in LL-37 resistance was further supported by testing the sensitivity of D39 $\Delta$ dltD and $\Delta l i c D 2$ deletion mutants towards the peptide. As expected from the literature [46], the $\Delta d l t D$ strain was more susceptible to LL-37. Interestingly, the growth of the stressed $\Delta l i c D 2$ mutant was even more reduced, indicating a role of the phosphorylcholine transferases LicD1-2 in LL-37 resistance, potentially by modifying the bacterial surface charge [24].

In conclusion, our study suggests that pneumococci may use adaptation mechanisms repulsion, export, and potentially degradation and interception to reduce the effects of the antimicrobial peptide LL-37. Certainly, this has yet to be confirmed in follow up experiments. However, as many of the presented candidates are changed at transcriptome and proteome levels in pneumococci, and several sequence similar proteins were already linked to AMP resistance in other bacteria, the presented candidates should be considered as highly relevant drug targets.

Finally, global knowledge about bacterial resistance mechanisms to AMPs could be used to design new drugs, blocking the functional players of resistance. These components would sensitize pathogens to already existing AMPs in the human body. In fact, it was already shown that inhibition of the dlt operon, normally reducing the bacterial membrane charge by modifying teichoic acids with D-alanine, sensitizes Gram-positive B. subtilis to cell-wall acting-antibiotics [56]. Other targets for this approach could be the teichoic acid flippase TacF (SPD_1128), the phosphorylcholine transferases LicD1-2 or stress-induced efflux pumps that potentially export AMPs out of the bacteria. However, further research is needed to verify the role of AMP resistance candidates discussed here and design appropriate inhibitor molecules.

Supplementary Materials: The following are available online at http://www.mdpi.com/2076-2607/8/3/413/s1.

Author Contributions: Conceptualization, S.M. and D.B.; methodology, P.-A.M.; formal analysis, P.-A.M.; investigation, P.-A.M.; resources, T.P.K. and S.H.; writing-original draft preparation, P.-A.M. and T.P.K.; writing-review and editing, S.M., S.H., D.B.; visualization, P.-A.M.; supervision, D.B.; project administration, S.M.; funding acquisition, S.H. and D.B. All authors have read and agreed to the published version of the manuscript. 
Funding: This work was funded by the Deutsche Forschungsgemeinschaft within the Research Training Group 1870 (DFG GRK 1870).

Acknowledgments: We want to thank Stephanie Hirschmann for valuable input on cultivation of pneumococci, Nathalie Heß for construction of the pneumococcal licD2 mutant and Anne Ostrzinski for technical assistance.

Conflicts of Interest: The authors declare no conflict of interest. The funders had no role in the design of the study; in the collection, analyses, or interpretation of data; in the writing of the manuscript, or in the decision to publish the results.

\section{References}

1. WHO. The Top 10 Causes of Death. Available online: http://www.who.int/mediacentre/factsheets/fs310/en/ (accessed on 14 November 2019).

2. WHO. Pneumonia. Available online: http://www.who.int/mediacentre/factsheets/fs331/en/ (accessed on 14 November 2019).

3. Weiser, J.N.; Ferreira, D.M.; Paton, J.C. Streptococcus pneumoniae: transmission, colonization and invasion. Nat. Rev. Microbiol. 2018, 16, 355-367. [CrossRef] [PubMed]

4. Ladhani, S.N.; Collins, S.; Djennad, A.; Sheppard, C.L.; Borrow, R.; Fry, N.K.; Andrews, N.J.; Miller, E.; Ramsay, M.E. Rapid increase in non-vaccine serotypes causing invasive pneumococcal disease in England and Wales, 2000-17: A prospective national observational cohort study. Lancet Infect. Dis. 2018, 18, 441-451. [CrossRef]

5. WHO. WHO Publishes List of Bacteria for Which New Antibiotics Are Urgently Needed. Available online: http://www.who.int/mediacentre/news/releases/2017/bacteria-antibiotics-needed/en/ (accessed on 14 November 2019).

6. Beisswenger, C.; Bals, R. Antimicrobial peptides in lung Inflammation. In Mechanisms of Epithelial Defense; Kabelitz, D., Schröder, J.-M., Eds.; Karger: Basel, Switzerland, 2005; Volume 86, pp. 55-71.

7. Yeaman, M.R.; Yount, N.Y. Mechanisms of antimicrobial peptide action and resistance. Pharm. Rev. 2003, 55, 27-55. [CrossRef] [PubMed]

8. Lai, Y.; Gallo, R.L. AMPed up immunity: how antimicrobial peptides have multiple roles in immune defense. Trends Immunol. 2009, 30, 131-141. [CrossRef]

9. Peschel, A.; Sahl, H.G. The co-evolution of host cationic antimicrobial peptides and microbial resistance. Nat. Rev. Microbiol. 2006, 4, 529-536. [CrossRef]

10. Vandamme, D.; Landuyt, B.; Luyten, W.; Schoofs, L. A comprehensive summary of LL-37, the factotum human cathelicidin peptide. Cell. Immunol. 2012, 280, 22-35. [CrossRef]

11. Malanovic, N.; Lohner, K. Antimicrobial peptides targeting Gram-positive bacteria. Pharmaceuticals 2016, 9, 59. [CrossRef]

12. Zasloff, M. Antimicrobial peptides of multicellular organisms. Nature 2002, 415, 389-395. [CrossRef]

13. Peters, B.M.; Shirtliff, M.E.; Jabra-Rizk, M.A. Antimicrobial peptides: primeval molecules or future drugs? PLoS Pathog. 2010, 6. [CrossRef]

14. Tjabringa, G.S.; Rabe, K.F.; Hiemstra, P.S. The human cathelicidin LL-37: a multifunctional peptide involved in infection and inflammation in the lung. Pulm. Pharm. 2005, 18, 321-327. [CrossRef]

15. Hiemstra, P.S.; Amatngalim, G.D.; van der Does, A.M.; Taube, C. Antimicrobial peptides and innate lung defenses: role in infectious and noninfectious lung diseases and therapeutic applications. Chest 2016, 149, 545-551. [CrossRef] [PubMed]

16. Kietzman, C.C.; Gao, G.; Mann, B.; Myers, L.; Tuomanen, E.I. Dynamic capsule restructuring by the main pneumococcal autolysin LytA in response to the epithelium. Nat. Commun. 2016, 7. [CrossRef] [PubMed]

17. Henzler Wildman, K.A.; Lee, D.-K.; Ramamoorthy, A. Mechanism of lipid bilayer disruption by the human antimicrobial peptide, LL-37. Biochemistry 2003, 42, 6545-6558. [CrossRef]

18. Brogden, K.A. Antimicrobial peptides: pore formers or metabolic inhibitors in bacteria? Nat. Rev. Microbiol. 2005, 3, 238-250. [CrossRef] [PubMed]

19. Lee, C.C.; Sun, Y.; Qian, S.; Huang, H.W. Transmembrane pores formed by human antimicrobial peptide LL-37. Biophys. J. 2011, 100, 1688-1696. [CrossRef] [PubMed]

20. Le, C.F.; Fang, C.M.; Sekaran, S.D. Intracellular targeting mechanisms by antimicrobial peptides. Antimicrob. Agents Chemother. 2017, 61. [CrossRef] 
21. Dürr, U.H.; Sudheendra, U.S.; Ramamoorthy, A. LL-37, the only human member of the cathelicidin family of antimicrobial peptides. Biochim. Biophys. Acta 2006, 1758, 1408-1425. [CrossRef]

22. LaRock, C.N.; Nizet, V. Cationic antimicrobial peptide resistance mechanisms of streptococcal pathogens. Biochim. Biophys. Acta 2015, 1848, 3047-3054. [CrossRef]

23. Koprivnjak, T.; Peschel, A. Bacterial resistance mechanisms against host defense peptides. Cell. Mol. Life Sci. 2011, 68, 2243-2254. [CrossRef]

24. Lysenko, E.S.; Gould, J.; Bals, R.; Wilson, J.M.; Weiser, J.N. Bacterial phosphorylcholine decreases susceptibility to the antimicrobial peptide LL-37/hCAP18 expressed in the upper respiratory tract. Infect. Immun. 2000, 68, 1664-1671. [CrossRef]

25. Roy, H.; Dare, K.; Ibba, M. Adaptation of the bacterial membrane to changing environments using aminoacylated phospholipids. Mol. Microbiol. 2009, 71, 547-550. [CrossRef] [PubMed]

26. Hamilton, A.; Popham, D.L.; Carl, D.J.; Lauth, X.; Nizet, V.; Jones, A.L. Penicillin-binding protein 1a promotes resistance of group B streptococcus to antimicrobial peptides. Infect. Immun. 2006, 74, 6179-6187. [CrossRef] [PubMed]

27. Scott, M.G.; Gold, M.R.; Hancock, R.E.W. Interaction of cationic peptides with lipoteichoic acid and Gram-positive bacteria. Infect. Immun. 1999, 67, 6445-6453. [PubMed]

28. Zähner, D.; Zhou, X.; Chancey, S.T.; Pohl, J.; Shafer, W.M.; Stephens, D.S. Human antimicrobial peptide LL-37 induces MefE/Mel-mediated macrolide resistance in Streptococcus pneumoniae. Antimicrob. Agents Chemother. 2010, 54, 3516-3519. [CrossRef] [PubMed]

29. Li, M.; Lai, Y.; Villaruz, A.E.; Cha, D.J.; Sturdevant, D.E.; Otto, M. Gram-positive three-component antimicrobial peptide-sensing system. Proc. Natl. Acad. Sci. USA 2007, 104, 9469-9474. [CrossRef] [PubMed]

30. Elbourne, L.D.; Tetu, S.G.; Hassan, K.A.; Paulsen, I.T. TransportDB 2.0: a database for exploring membrane transporters in sequenced genomes from all domains of life. Nucleic Acids Res. 2017, 45, D320-D324. [CrossRef]

31. Cole, J.N.; Nizet, V. Bacterial evasion of host antimicrobial peptide defenses. Microbiol. Spectr. $2016,4$. [CrossRef]

32. Wang, G.; Li, X.; Wang, Z. APD3: the antimicrobial peptide database as a tool for research and education. Nucleic Acids Res. 2016, 44, D1087-D1093. [CrossRef]

33. Nuding, S.; Frasch, T.; Schaller, M.; Stange, E.F.; Zabel, L.T. Synergistic effects of antimicrobial peptides and antibiotics against Clostridium difficile. Antimicrob. Agents Chemother. 2014, 58, 5719-5725. [CrossRef]

34. Lazar, V.; Martins, A.; Spohn, R.; Daruka, L.; Grezal, G.; Fekete, G.; Szamel, M.; Jangir, P.K.; Kintses, B.; Csorgo, B.; et al. Antibiotic-resistant bacteria show widespread collateral sensitivity to antimicrobial peptides. Nat. Microbiol. 2018, 3, 718-731. [CrossRef]

35. Schulz, C.; Gierok, P.; Petruschka, L.; Lalk, M.; Mader, U.; Hammerschmidt, S. Regulation of the arginine deiminase system by ArgR2 interferes with arginine metabolism and fitness of Streptococcus pneumoniae. mBio 2014, 5. [CrossRef]

36. Cold Spring Harbor Laboratory Press. Phosphate-buffered saline (PBS). Available online: http://cshprotocols. cshlp.org/content/2006/1/pdb.rec8247 (accessed on 14 November 2019).

37. Saleh, M.; Bartual, S.G.; Abdullah, M.R.; Jensch, I.; Asmat, T.M.; Petruschka, L.; Pribyl, T.; Gellert, M.; Lillig, C.H.; Antelmann, H.; et al. Molecular architecture of Streptococcus pneumoniae surface thioredoxin-fold lipoproteins crucial for extracellular oxidative stress resistance and maintenance of virulence. Embo Mol. Med. 2013, 5, 1852-1870. [CrossRef] [PubMed]

38. Yang, F.; Shen, Y.; Camp, D.G., 2nd; Smith, R.D. High-pH reversed-phase chromatography with fraction concatenation for 2D proteomic analysis. Expert Rev. Proteom. 2012, 9, 129-134. [CrossRef] [PubMed]

39. Cox, J.; Neuhauser, N.; Michalski, A.; Scheltema, R.A.; Olsen, J.V.; Mann, M. Andromeda: A peptide search engine integrated into the MaxQuant environment. J. Proteome Res. 2011, 10, 1794-1805. [CrossRef] [PubMed]

40. Tyanova, S.; Temu, T.; Sinitcyn, P.; Carlson, A.; Hein, M.Y.; Geiger, T.; Mann, M.; Cox, J. The Perseus computational platform for comprehensive analysis of (prote)omics data. Nat. Methods 2016, 13, 731-740. [CrossRef] [PubMed]

41. Slager, J.; Aprianto, R.; Veening, J.W. Deep genome annotation of the opportunistic human pathogen Streptococcus pneumoniae D39. Nucleic Acids Res. 2018, 46, 9971-9989. [CrossRef] 
42. Peabody, M.A.; Laird, M.R.; Vlasschaert, C.; Lo, R.; Brinkman, F.S. PSORTdb: Expanding the bacteria and archaea protein subcellular localization database to better reflect diversity in cell envelope structures. Nucleic Acids Res. 2016, 44, D663-D668. [CrossRef]

43. Halfmann, A.; Kovacs, M.; Hakenbeck, R.; Bruckner, R. Identification of the genes directly controlled by the response regulator CiaR in Streptococcus pneumoniae: Five out of 15 promoters drive expression of small non-coding RNAs. Mol. Microbiol. 2007, 66, 110-126. [CrossRef]

44. Perez-Riverol, Y.; Csordas, A.; Bai, J.; Bernal-Llinares, M.; Hewapathirana, S.; Kundu, D.J.; Inuganti, A.; Griss, J.; Mayer, G.; Eisenacher, M.; et al. The PRIDE database and related tools and resources in 2019: Improving support for quantification data. Nucleic Acids Res. 2019, 47, D442-D450. [CrossRef]

45. Majchrzykiewicz, J.A.; Kuipers, O.P.; Bijlsma, J.J. Generic and specific adaptive responses of Streptococcus pneumoniae to challenge with three distinct antimicrobial peptides, bacitracin, LL-37, and nisin. Antimicrob. Agents Chemother. 2010, 54, 440-451. [CrossRef]

46. Kovacs, M.; Halfmann, A.; Fedtke, I.; Heintz, M.; Peschel, A.; Vollmer, W.; Hakenbeck, R.; Bruckner, R. A functional $d l t$ operon, encoding proteins required for incorporation of D-alanine in teichoic acids in Gram-positive bacteria, confers resistance to cationic antimicrobial peptides in Streptococcus pneumoniae. J. Bacteriol. 2006, 188, 5797-5805. [CrossRef] [PubMed]

47. Zhang, J.-R.; Idanpaan-Heikkila, I.; Fischer, W.; Tuomanen, E.I. Pneumococcal licD2 gene is involved in phosphorylcholine metabolism. Mol. Microbiol. 1999, 31, 1477-1488. [CrossRef] [PubMed]

48. Damjanovic, M.; Kharat, A.S.; Eberhardt, A.; Tomasz, A.; Vollmer, W. The essential tacF gene is responsible for the choline-dependent growth phenotype of Streptococcus pneumoniae. J. Bacteriol. 2007, 189, 7105-7111. [CrossRef]

49. Waldow, F.; Kohler, T.P.; Hess, N.; Schwudke, D.; Hammerschmidt, S.; Gisch, N. Attachment of phosphorylcholine residues to pneumococcal teichoic acids and modification of substitution patterns by the phosphorylcholine esterase. J. Biol. Chem. 2018, 293, 10620-10629. [CrossRef] [PubMed]

50. Collins, B.; Curtis, N.; Cotter, P.D.; Hill, C.; Ross, R.P. The ABC transporter AnrAB contributes to the innate resistance of Listeria monocytogenes to nisin, bacitracin, and various beta-lactam antibiotics. Antimicrob. Agents Chemother. 2010, 54, 4416-4423. [CrossRef] [PubMed]

51. Staron, A.; Finkeisen, D.E.; Mascher, T. Peptide antibiotic sensing and detoxification modules of Bacillus subtilis. Antimicrob. Agents Chemother. 2011, 55, 515-525. [CrossRef]

52. Nawrocki, K.L.; Crispell, E.K.; McBride, S.M. Antimicrobial peptide resistance mechanisms of Gram-positive bacteria. Antibiotics 2014, 3, 461-492. [CrossRef]

53. Gomez-Mejia, A.; Gamez, G.; Hammerschmidt, S. Streptococcus pneumoniae two-component regulatory systems: the interplay of the pneumococcus with its environment. Int. J. Med. Microbiol. 2017, 308, 722-737. [CrossRef]

54. Melin, M.; Di Paolo, E.; Tikkanen, L.; Jarva, H.; Neyt, C.; Kayhty, H.; Meri, S.; Poolman, J.; Vakevainen, M. Interaction of pneumococcal histidine triad proteins with human complement. Infect. Immun. 2010, 78, 2089-2098. [CrossRef]

55. Standish, A.J.; Whittall, J.J.; Morona, R. Tyrosine phosphorylation enhances activity of pneumococcal autolysin LytA. Microbiology 2014, 160, 2745-2754. [CrossRef]

56. May, J.J.; Finking, R.; Wiegeshoff, F.; Weber, T.T.; Bandur, N.; Koert, U.; Marahiel, M.A. Inhibition of the D-alanine:D-alanyl carrier protein ligase from Bacillus subtilis increases the bacterium's susceptibility to antibiotics that target the cell wall. FEBS J. 2005, 272, 2993-3003. [CrossRef] [PubMed]

(C) 2020 by the authors. Licensee MDPI, Basel, Switzerland. This article is an open access article distributed under the terms and conditions of the Creative Commons Attribution (CC BY) license (http://creativecommons.org/licenses/by/4.0/). 